\title{
RISCO DA DENGUE NOS MUNICÍPIOS PANTANEIROS DE MATO GROSSO, BRASIL
}

\author{
RISK OF DENGUE IN MUNICIPALITIES PANTANAL OF MATO \\ GROSSO, BRAZIL
}

\author{
EL RIESGO DE DENGUE EN LOS MUNICIPIOS DEL PANTANAL \\ DE MATO GROSSO, BRASIL
}

Rosilainy Surubi Fernandes - Universidade do Estado de Mato Grosso - Cáceres - Mato Grosso - Brasil

rosilainysf@gmail.com

Sandra Mara Alves da Silva Neves - Universidade do Estado de Mato Grosso - Cáceres - Mato Grosso - Brasil ssneves@unemat.br

Ronaldo José Neves - Universidade do Estado de Mato Grosso - Cáceres - Mato Grosso - Brasil rjneves@terra.com.br

\section{Resumo}

Este artigo tem o objetivo de analisar o risco de transmissão da dengue, por meio do modelo tempo-espacial, nos municípios do Pantanal Mato-Grossense. Foram considerados os casos de dengue confirmados e autóctones registrados no período de 2009 a 2011 no Sistema de Informação de Agravos de Notificação (Sinan) da Vigilância epidemiológica de Cuiabá/MT e na Secretaria Regional de Saúde de Cáceres/MT. Estas três medidas de risco tempo-espacial foram utilizadas: frequência, duração e intensidade, operacionalizadas no software ArcGis 9.2, da Esri. 0 índice de Moran Global e 0 índice de Moran Local foram usados para avaliar a autocorrelação espacial dos três índices. No período estudado, os municípios de Cáceres, Poconé, Santo Antônio do Leverger e de Nossa Senhora do Livramento apresentaram os maiores índices de frequência, duração e intensidade; contudo, Cáceres foi o que apresentou maiores riscos da endemia. 0 índice de Moran Global apresentou baixa correlação e 0 índice de Moran Local apresentou aglomerados em alguns municípios, de acordo com o índice utilizado. Estes índices podem ser úteis nas tomadas de decisão das autoridades e da saúde pública.

Palavras-chave: geotecnologias, geossaúde, epidemiologia, bioma Pantanal.

\section{Abstract}

The aim of this study is to analyze the risk of transmission, via time/spatial model, of the confirmed cases of dengue in the municipalities belonging to the Pantanal of Mato Grosso. We considered the cases of dengue confirmed and the original inhabitant recorded in the period from 2009 to 2011 at the Information System for Notifiable Diseases (SINAN) in the epidemiological Vigilance - Cuiabá and the Cáceres Regional Health. Three measures of risk time/spatial were used: frequency, duration and intensity, managed in the software ArcGIS 9.2, ESRI. Moran's Global and Local index were used to evaluate the spatial autocorrelation of the three index. In the period studied, the cities of Cáceres, Poconé, Santo Antônio de Leverger and Nossa Senhora do Livramento presented the highest rates of frequency, duration and intensity, however, Cáceres showed the highest risk of the disease. Moran's Global Index showed low correlation and Moran's Local Index showed clusters in some cities according to the index used. These indexes may be useful in the authorities decision and public health.

Key words: geo, geohealth, epidemiology, Pantanal biome. 


\section{Resumen}

Este estudio tuvo como objetivo, analizar el riesgo de transmisión del dengue a partir de casos confirmados en los municipios pertenecientes al Pantanal del estado de Mato Grosso, utilizando un modelo temporo-espacial. Considerando los casos confirmados de dengue autóctono registrados en el período de 2009 a 2011 por el Sistema de Información de Casos Graves de Notificación (SINAN), perteneciente a la Vigilancia Epidemiológica de la ciudad de Cuiabá y a la Secretaría Regional de Salud de Cáceres, fueron aplicadas tres medidas de riesgo temporo-espacial: frequencia, duración e intensidad, operacionalizadas en el software ArcGis 9.2. Los índices Moran Global y Moran Local se utilizaron para evaluar la autocorrelación espacial de los tres índices. Como resultado se evidenció que, en el período estudiado, los municipios de Cáceres, Poconé, Santo Antônio do Leverger y Nossa Senhora do Livramento presentaron los mayores índices de frequencia, duración e intensidad, siendo Cáceres el de mayores riesgos de epidemia. El índice de Moran Global presentó una baja correlación y el de Moran Local presentó aglomerados en algunos municipios. Estos índices pueden ser útiles en la toma de decisiones de las autoridades de salud pública

Palabras clave: geo, geosaúde, epidemiología, bioma Pantanal.

\section{Introdução}

O mapeamento de doenças vem sendo um instrumento básico no campo da saúde pública. De acordo com Siqueira-Junior et al. (2008), desde a década de 1990, as técnicas de análise de dados têm sido apuradas para gerar mapas de identificação de áreas de risco.

A espacialização de regiões de maiores infecções, realizada através dos Sistemas de Informações Geográficas (SIG), auxilia na prevenção de endemias e orienta as autoridades em saúde pública sobre os problemas ambientais e de saúde de diferentes localidades.

Os cenários de saúde desenhados pelas técnicas de georreferenciamento agregam, entre outros, dados ambientais e estruturais da saúde ao componente geográfico da relação de vizinhança entre as diferentes áreas (Mukai; Alves; Nascimento, 2009).

O mapeamento de casos de doença fornece uma visão deles no espaço, entretanto, não pode confirmar estatisticamente os agrupamentos ou autocorrelações espaciais. Para isso, a estatística espacial é extensivamente utilizada para relacionar valores de casos e sua localização geográfica (Croner; Sperling; Broome, 1996). O índice de Moran Global e o índice de Moran Local são técnicas da geoestatística baseadas no conceito de autocorrelação espacial, aplicáveis aos objetos espaciais com área definida e atributos numéricos associados, como casos de endemias ocorrentes em uma localidade (Marques, 2009).

Entre as diversas doenças de importância médica, a dengue tem causado preocupação às autoridades da saúde pública em virtude da am- 
pla distribuição do vetor e a possibilidade de causar casos graves e letais, como a febre hemorrágica.

Segundo Galli e Chiaravalloti Neto (2008), no Brasil, o estado endêmico da doença já alterou os indicadores de morbidade e a amplitude dessas incidências, nos últimos anos, ultrapassou a de todas as outras doenças de notificação compulsória.

O estado de Mato Grosso, de acordo com o Informe Epidemiológico da Dengue (Brasil, 2010), dentre a Região Centro-Oeste, apresenta um dos maiores índices da doença. Dentro deste espaço, o bioma Pantanal apresenta uma diversidade biológica decorrente de múltiplas e complexas interações. Somada ainda à biodiversidade física, há peculiaridades demográficas com a presença de pantaneiros, povos indígenas, populações de fronteira da Bolívia, do Paraguai e de outros países latino-americanos. Dessa forma, Cunha e Atanaka-Santos (2011) mencionam a carência de pesquisas que busquem (re)conhecer a situação de saúde e a ocorrência de agentes causadores de doenças que são decorrentes de contatos com ambientes que podem apresentar vulnerabilidade e abrigar reservatórios de doenças, especialmente aquelas transmitidas por vetores, como a dengue.

Nesse contexto, alguns estudos epidemiológicos sobre a dengue pautam-se nas taxas de incidências que acabam gerando resultados limitados. Por isso, Wen et al. (2006) propuseram um modelo de risco tempo-espacial, para o mapeamento da distribuição geográfica de casos, que baseia-se em três medidas de risco temporal no espaço geográfico - frequência, duração e intensidade - consideradas adequadas para a identificação de áreas de risco.

A combinação dos três indicadores é fundamental para diferenciar as características de risco, a definição da frequência de ocorrência da endemia em determinado período e a duração em semanas epidemiológicas, ou seja, a persistência da doença e a intensidade que é caracterizada pela severidade da dengue apontam padrões da epidemia em diferentes áreas.

Diante disso, este estudo tem o objetivo de analisar o risco de transmissão, via modelo tempo-espacial, dos casos de dengue confirmados nos municípios pertencentes ao Pantanal Mato-Grossense. 
Material e métodos

Área de estudo

A área de estudo compreende oito municípios que compõem o bioma Pantanal de Mato Grosso e que pertencem à Bacia do Alto Paraguai, localizada entre as latitudes $15^{\circ} 30^{\prime}$ e $22^{\circ} 30^{\prime}$ Sul e longitudes $54^{\circ} 45^{\prime}$ e $58^{\circ}$ 30' Oeste, sendo estes, Barão de Melgaço, Cáceres, Curvelândia, Itiquira, Lambari D' Oeste, Nossa Senhora do Livramento, Poconé e Santo Antônio do Leverger (Figura 1).

Esses municípios localizam-se na região sudoeste do estado e apresentam algumas características que os distinguem de outros municípios, especialmente quando se considera o número populacional, a densidade demográfica, o Produto Interno Bruto (PIB) entre outros fatores (Tabela 1).

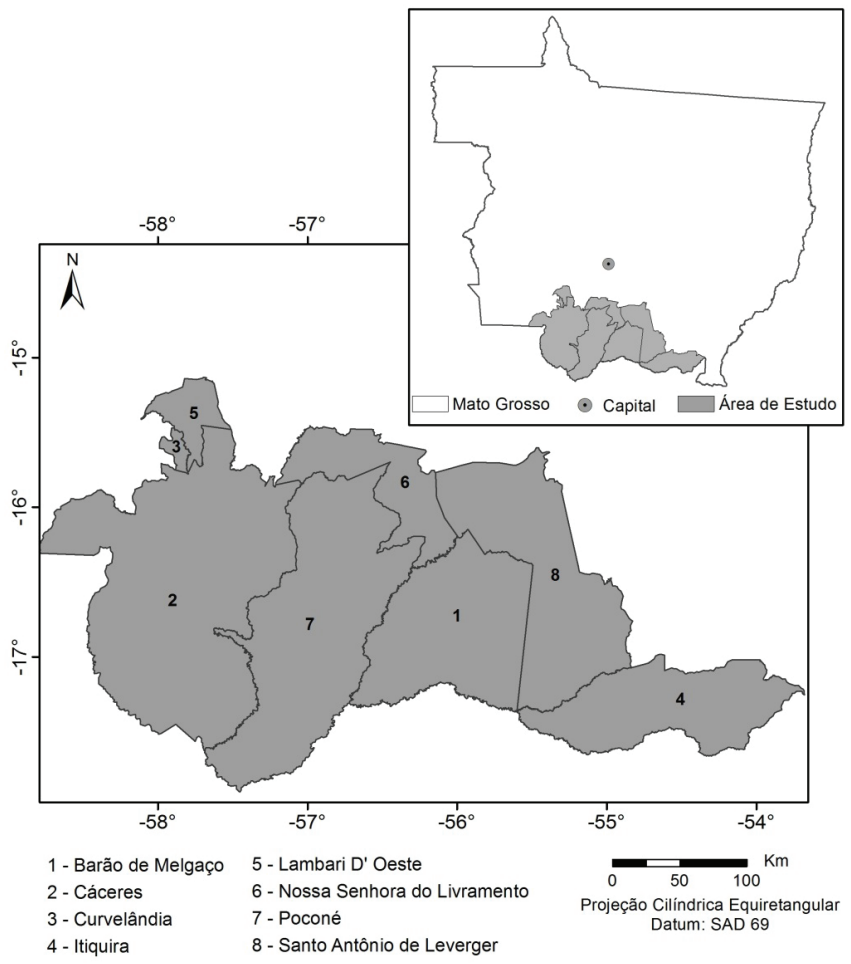

Figura 1: Localização dos municípios pantaneiros do estado de Mato Grosso. 
Tabela 1: Características populacionais dos municípios pantaneiros de Mato Grosso.

\begin{tabular}{|l|r|r|r|r|c|}
\hline Município & Homens & Mulheres & $\begin{array}{c}\text { Densidade } \\
\text { demográfica }\end{array}$ & $\begin{array}{c}\text { Taxa } \\
\text { alfabetização* }\end{array}$ & PIB \\
\hline Barão de Melgaço & 4.134 & 3.457 & 0,67 & 93,61 & $7.582,79$ \\
\hline Cáceres & 44.098 & 43.844 & 3,61 & 98,21 & $9.772,28$ \\
\hline Curvelândia & 2.534 & 2.332 & 13,53 & 93,05 & $7.410,50$ \\
\hline Itiquira & 6.225 & 5.253 & 1,32 & 100 & $43.538,35$ \\
\hline Lambari D’Oeste & 2.852 & 2.579 & 3,08 & 93,54 & $15.284,68$ \\
\hline $\begin{array}{l}\text { Nossa Senhora do } \\
\text { Livramento }\end{array}$ & 6.270 & 5.339 & 2,10 & 84,35 & $7.673,86$ \\
\hline Poconé & 16.519 & 15.260 & 1,84 & 90,22 & $8.819,09$ \\
\hline $\begin{array}{l}\text { Santo Antônio do } \\
\text { Leverger }\end{array}$ & 9.861 & 8.602 & 1,57 & 90,68 & $9.334,91$ \\
\hline
\end{tabular}

*População residente de 10 anos ou mais. Fonte: IBGE, 2010. Org.: Os autores, 2012.

\section{Procedimentos metodológicos}

Para este estudo, foram considerados os casos de dengue confirmados e autóctones registrados nestes municípios do bioma Pantanal, no estado de Mato Grosso: Barão de Melgaço, Cáceres, Curvelândia, Itiquira, Lambari D’ Oeste, Nossa Senhora do Livramento, Poconé e Santo Antônio do Leverger, entre 2009 e 2011. Para a confirmação dos casos, houve exame laboratorial e clínico-epidemiológico.

Os dados foram obtidos através do banco de dados do Sistema de Informação de Agravos de Notificação (Sinan) da Vigilância epidemiológica de Cuiabá/MT e da Secretaria Regional de Saúde de Cáceres/MT.

Para avaliar o risco de transmissão através do modelo tempo-espacial proposto por Wen et al. (2006), foram adotados três índices de risco temporal, calculados para cada unidade espacial e ano sazonal, no software ArcGis, com base nas informações contidas no banco de dados geográficos:

\section{- Índice de frequência $(\alpha), \alpha=S E / S T$}

ST é o número total de semanas durante o período considerado e SE, o número total de semanas com um ou mais casos ocorridos durante o período considerado. 
Isso representa a proporção de semanas com um ou mais casos ou a probabilidade de que um ou mais casos ocorram em uma determinada semana no período estudado (um ano ou 52 semanas).

Segundo Wen et al. (2006), o índice de frequência mais próximo a 1 apresenta maior possibilidade de ocorrência da dengue em determinadas semanas epidêmicas, no período estudado.

- Índice de duração $(\beta), \beta=\mathrm{SE} / \mathrm{OE}$

Nesse caso, SE é o número total de semanas com um ou mais casos ocorridos durante o período considerado e OE, o número total de ondas epidêmicas durante o período considerado.

Vale ressaltar que uma onda epidêmica é definida como um agrupamento de semanas com a ocorrência de casos sem interrupção. Esse índice aponta a persistência da transmissão da doença e representa a duração média, em semanas, das ondas epidêmicas que ocorreram no período considerado.

Os valores elevados indicam a duração da doença em semanas.

\section{- Índice de intensidade $(\gamma), \gamma=\mathrm{TI} / \mathrm{OE}$}

TI é a taxa de incidência durante o período considerado e OE, o número total de ondas epidêmicas durante o período considerado.

Assim, há a avaliação da severidade da transmissão, tendo como base a sequência de semanas com a ocorrência de casos sem interrupção. Valores altos significam que houve a transmissão mais concentrada no tempo.

Para a geração dos mapas temáticos representativos dos índices, no programa ArcGis, versão 9.2, da Esri, foram utilizados os valores dos três índices tempo-espaciais e a malha digital dos municípios, obtida no sítio do Instituto Brasileiro de Geografia e Estatística (IBGE).

Também foram usados o Índice de Moran Global e o Indicie de Moran Local, em que o primeiro testa se as áreas conectadas apresentam maior semelhança quanto ao indicador estudado do que o esperado em um padrão aleatório, variando de -1 a +1 . O grau de autocorrelação existente pode ser quantificado, sendo positivo para a correlação direta e negativo quando inversa.

O índice de Moran Local - Local Indicators of Spatial Association (LISA) - de casos de dengue permite encontrar os bairros com dependên- 
cia espacial não observados nos índices globais. Conforme Anselin (1995), na geração do LISA, é avaliada a significância dos valores do índice de Moran Local obtido para cada objeto (polígono) em relação à hipótese de não existência de autocorrelação espacial (hipótese nula). Esses valores são classificados em quatro grupos: não significantes (p-valor $>0,05)$; significância a 95\% (0,01 < p-valor $\leq$ 0,05); significância a 99\% $(0,001<$ p-valor $\leq$ 0,01); significância a 99,9\% (p-valor $\leq 0,001)$.

Avaliação de risco da transmissão de dengue nos municípios do Pantanal Mato-Grossense

No período estudado, foram contabilizados 4.987 casos autóctones de dengue nos municípios pesquisados. Em 2009, houve o registro dos maiores picos de casos de dengue, se comparado aos demais anos pesquisados (Figura 2); as semanas epidemiológicas 11, 12 e 13 apresentaram uma das maiores incidências, correspondentes ao mês de março, período de término das chuvas. As semanas 45 a 49, para 2009, concentraram elevado número de casos, e isso pode ter ocorrido em virtude do início do período de maior precipitação. As menores ocorrências estiveram na primeira semana e entre as semanas 22 e 24 .

Em 2010, as semanas entre 1 e 17 apresentaram altos índices de casos de dengue; no entanto, diferente do mês anterior, esses valores tiveram uma redução a partir da semana 18 e não se elevaram novamente, independente do período de chuvas.

Já no ano de 2011, as incidências de casos de dengue estiveram abaixo dos demais anos pesquisados. Possivelmente esse fato tem relação com as campanhas de prevenção da Secretaria de Saúde, em conjunto com a Vigilância Epidemiológica, ou com a imunização ocorrida em 2009, que serviu para protejer a população, ou seja, as pessoas que adquiriram a doença em anos anteriores poderiam apresentar imunidade para determinado sorotipo de dengue.

Os resultados desses três anos, nas áreas estudadas, indicam a influência dos períodos chuvosos e de estiagem quanto à presença ou ausência do vetor da dengue. Isso é confirmado através de pesquisas envolvendo casos de dengue e clima (Barbosa e Lourenço, 2010; Ribeiro et al., 2006; Glasser e Gomes, 2002; Forattini, 2002; Rebêlo, 1999). 


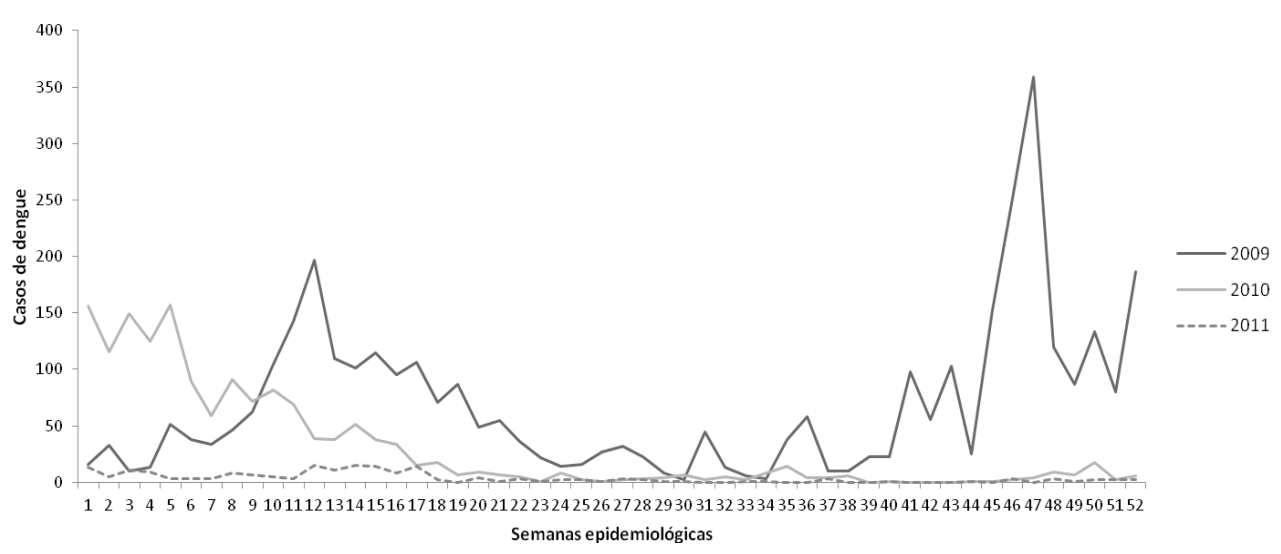

Figura 2: Casos de dengue por semana epidemiológica nos municípios do bioma Pantanal de Mato Grosso entre 2009 e 2010.

A utilização de mapas, para a espacialização dos três índices, auxilia no monitoramento rápido da dengue, pois permite uma melhor visualização dos aglomerados temporalmente definidos dos casos da doença. O mapeamento realizado com esse método, através do Sistema de Informação Geográfica (SIG), permite a inserção no banco de dados dos valores dos índices, calculados com base na semana epidemiológica, junto com a taxa de incidência de cada município, possibilitando, assim, a agilidade no acompanhamento da doença.

No ano de 2009, o município de Cáceres apresentou maiores valores dos índices de frequência $(\alpha=0,98)$, seguido de Poconé $(\alpha=0,576)$ e Lambari D'Oeste $(\alpha=0,423)$. Nesse caso, os valores indicaram que os municípios com maiores frequências apresentaram número maior de semanas que continham, pelo menos, um caso da doença (Figura 3). A duração da endemia esteve entre 0,0 e 25,50 semanas, concentrando-se, sobretudo, em Cáceres, Poconé e Santo Antônio de Leverger. A maior intensidade ocorreu no município de Cáceres $(\gamma=16,38)$, o que demonstra que nos demais municípios as ondas são mais curtas e com leve intensidade de epidemia de dengue (Figura 3). 


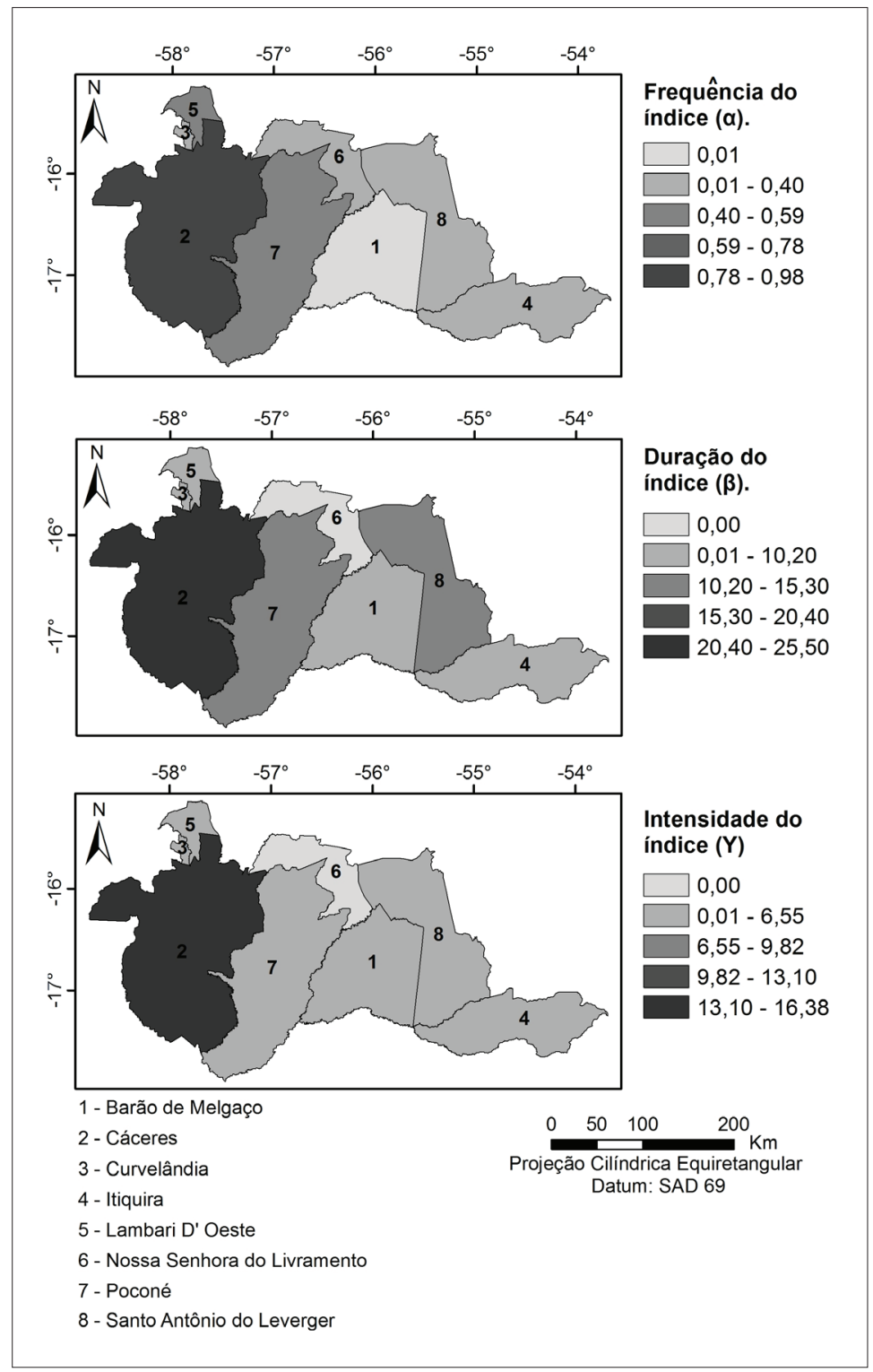

Figura 3: Distribuição tempo-espacial dos três índices de risco nos municípios do Pantanal Mato-Grossense em 2009. 
Em 2010, os maiores valores da frequência de casos da dengue ocorreram nos municípios de Cáceres, Nossa Senhora do Livramento e Itiquira ( $\alpha=0,903 ; \alpha=0,653$ e $\alpha=0,44,2$ respectivamente). O município de Barão de Melgaço não apresentou incidência da endemia nesse ano (Figura 4). A duração ocorreu entre 0 e 23,5 semanas e a extensão foi maior em Cáceres $(\beta=23,5)$ e Itiquira ( $\beta=23)$. Os valores altos de duração indicaram que os casos da doença têm menor possibilidade de desaparecer nesse período.

Os municípios de Cáceres e Itiquira também apresentaram alto grau de intensidade da doença em $2010(\gamma=16,3$ e $\gamma=18,29)$. A alta frequência, a intensidade e a baixa duração, no município de Nossa Senhora do Livramento, podem indicar a existência de pulsos de transmissão frequentemente interrompidos, e mostram que as medidas de controle não foram efetivas em longo prazo. No município de Itiquira, a duração e a intensidade foram maiores que a frequência. Segundo Galli e Chiaravalloti Neto (2008), os resultados obtidos de longa duração e a alta intensidade das áreas podem ser resultantes de uma transmissão severa, mas a baixa frequência aponta para o esgotamento de suscetíveis ou a relativa efetividade das medidas de combate ao vetor.

Em 2011, a incidência da dengue foi menor em comparação com os demais anos. Nossa Senhora do Livramento foi o município que apresentou maior frequência da endemia $(\alpha=0,269)$; duração $(\beta=14)$ e intensidade $(\gamma=8,09)$, seguido de Santo Antônio do Leverger $(\alpha=0,23 ; \beta=0,0 ; \gamma=$ 0,0). Observa-se que, no município de Santo Antônio do Leverger, ocorreu maior frequência; no entanto, menor duração e intensidade (Figura 5). Segundo Wen et al. (2006), a alta frequência, a baixa duração e a baixa intensidade das áreas podem estar associadas à existência de fontes de transmissão não identificadas.

Neste estudo, as características ambientais do município de Cáceres podem ter propiciado altos índices de frequência, duração e intensidade especialmente no ano de 2009. Souza (2010) considera que a elevada incidência de dengue nesse ano pode ser atribuída às altas temperaturas do local, entre outros fatores. De acordo com Forattini (2002), as condições climáticas caracterizadas pelas temperaturas elevadas, em geral, mostram relação positiva com a transmissão de dengue. Isso pode ser explicado porque a temperatura interfere na distribuição dos mosquitos, na frequência de suas picadas e no período de incubação do vírus, que varia de dez para sete dias, quando a temperatura passa de $27^{\circ} \mathrm{C}$ para $37^{\circ} \mathrm{C}$ (Mendonça, 2003). 


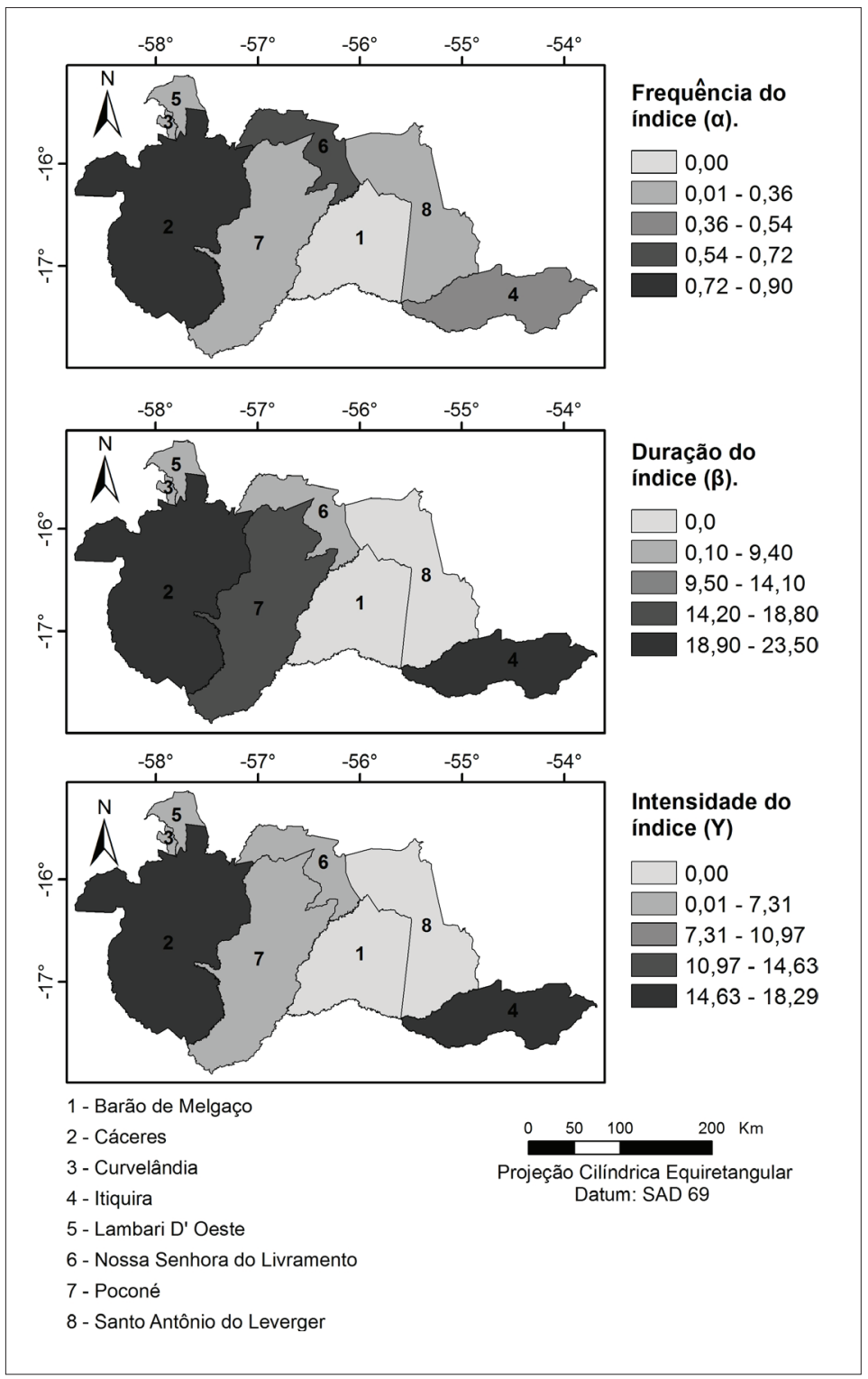

Figura 4: Distribuição tempo-espacial dos três índices de risco nos municípios do Pantanal Mato-Grossense em 2010. 


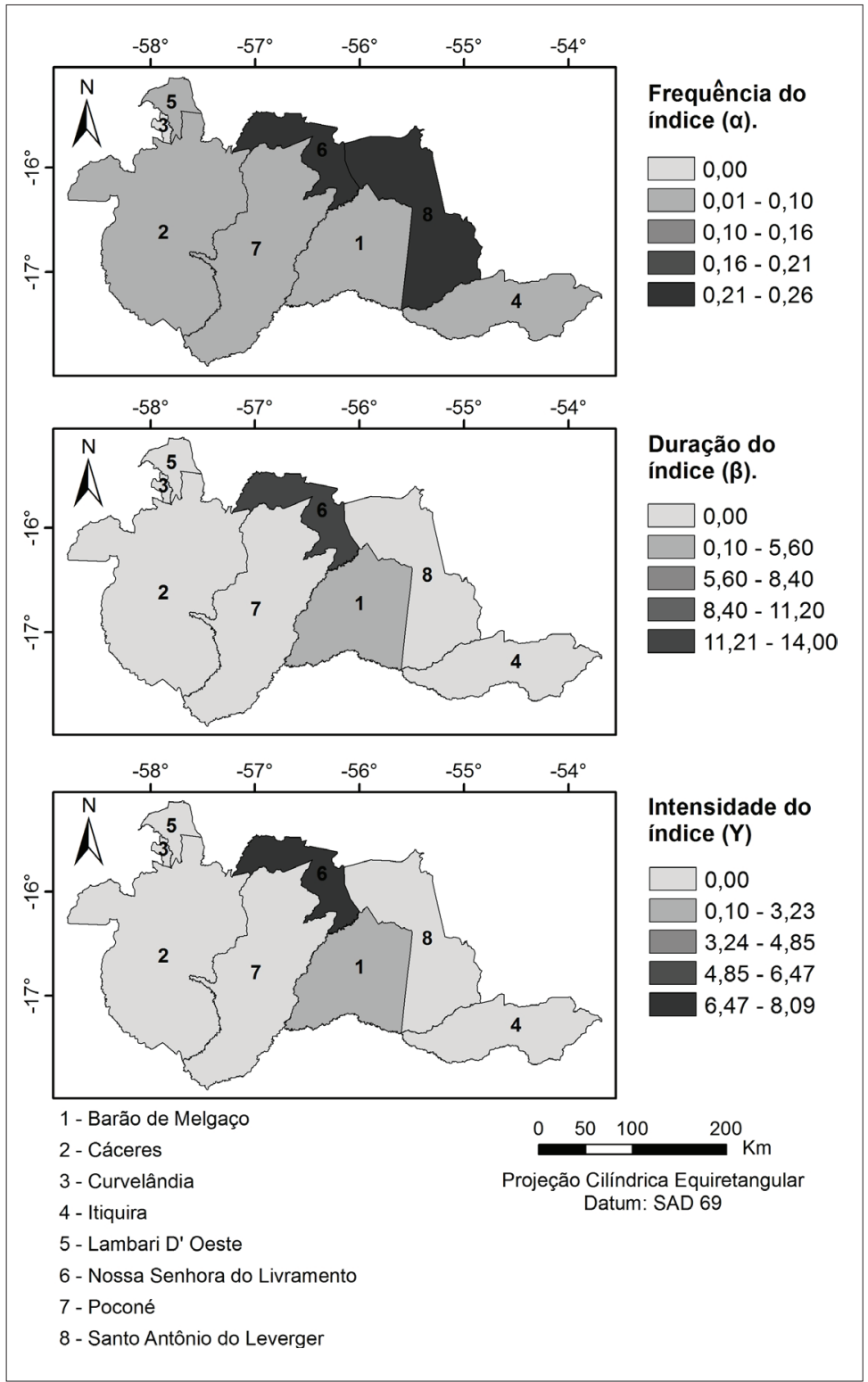

Figura 5: Distribuição tempo-espacial dos três índices de risco nos municípios do Pantanal Mato-Grossense em 2011. 
Ressalta-se que os municípios que apresentaram os maiores valores dos índices são os que possuem maior quantidade populacional. Isso confirma a relação do Aedes aegypti com ambientes urbanizados, fato constatado por Cox et al. (2007), que verificaram diferentes espécies de culicídios em distintas paisagens, e a predominância do Aedes aegypti em locais urbanizados e de elevada densidade populacional. De acordo com Teixeira et al. (2002), os centros urbanos são favorecedores da dispersão e do aumento da densidade do mosquito, considerando que o espaço social organizado influencia a interação entre o vetor, o vírus e o homem.

O índice de Moran Global para os três índices tempo-espaciais, em 2009, assinalou a fraca correlação espacial para o índice de frequência $(\alpha)$ $(I=0,109355 ; p=0,337)$, índice de duração $(\beta)(I=-0,276039 ; p=0,19)$ e índice de intensidade $(\gamma)(\mathrm{I}=-0,0633951 ; \mathrm{p}=0,44)$. Isso indica que existe pouca ou nenhuma semelhança quanto aos casos de dengue entre os municípios estudados, ou seja, cada município apresenta diferentes padrões para a transmissão dessa doença.

Entretanto, nos três anos pesquisados, a autocorrelação de Moran Local indicou que, no ano de 2009, muitos municípios apresentaram correlação para os três índices (Figura 6). Em 2009, quanto à frequência ( $\alpha$ ), o município de Barão de Melgaço apresentou 99,9\% de significância. Isso indica uma dependência desse município com relação à proporção de casos de dengue.

A duração $(\beta)$ apresentou significância para Nossa Senhora do Livramento e a intensidade $(\gamma)$ para o LISA foi significativo: 95\% para os municípios de Lambari D’ Oeste e Santo Antônio de Leverger e 99,9\% em Barão de Melgaço. Por isso, ressalta-se atenção para esses municípios, visto que a severidade da doença, nos dois últimos municípios, formou um agrupamento para a intensidade de casos de dengue, ou seja, essas áreas apresentam a transmissão da doença mais concentrada no tempo.

Os anos de 2010 e 2011 apresentaram fraca correlação espacial em muitos municípios; no entanto, alguns apresentaram significância como Itiquira com 99,9\% para os índices de duração e intensidade no ano de 2010 e Barão de Melgaço para intensidade em 2011, com 99,9\%. 


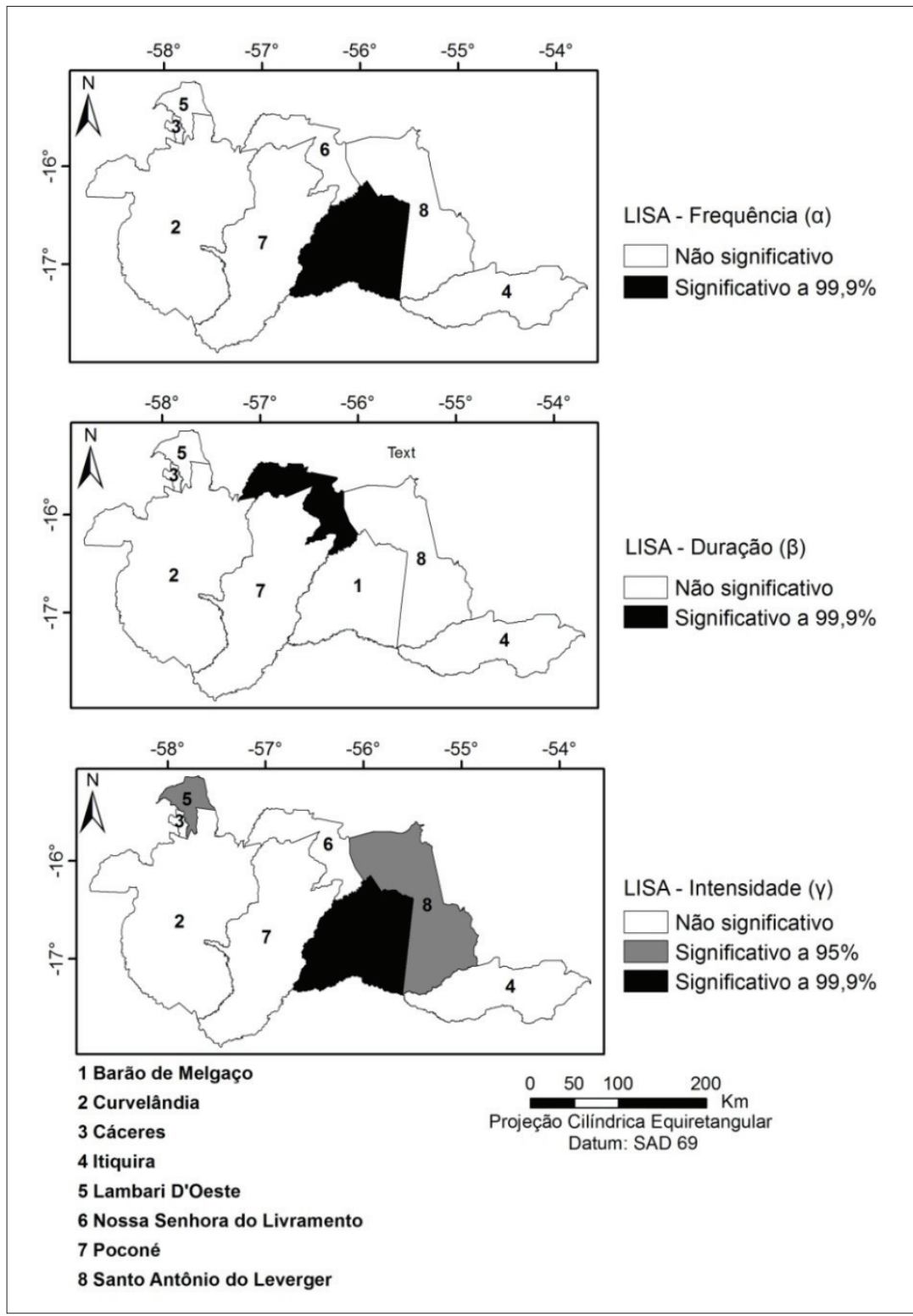

Figura 6: Autocorrelação dos três índices tempo-espaciais nos municípios pantaneiros de Mato Grosso em 2009. 
Vale destacar que, nos municípios onde os valores dos índices se mostraram baixos, não se deve dispensar atenção dos serviços de saúde, pois alterações no ambiente podem modificar os padrões de ocorrência da dengue.

As limitações deste estudo são as mesmas referentes à utilização de dados secundários, no entanto, como foram empregados dados autóctones e confirmados, eles não serão superestimados, como é comum nos modelos.

Os índices utilizados neste trabalho podem fornecer aos profissionais da saúde os níveis de risco, tendo em vista as características espaciais e temporais, ou seja, os locais e as semanas epidemiológicas. Essa abordagem pode ainda ser utilizada em pesquisas de outras doenças infecciosas e associada com os recursos geotecnológicos.

\section{Considerações finais}

O uso dos índices de risco (frequência, duração e intensidade) possibilitou a identificação e hierarquização dos municípios de Mato Grosso, contidos no bioma Pantanal, de maior risco para a ocorrência de dengue nos anos de 2009 e 2011. Destes, o município de Cáceres foi o que apresentou os maiores valores dos índices de frequência, duração e intensidade, seguido pelo de Poconé. Observou-se que, dependendo do ano analisado, o padrão é alterado, pois em 2011 Cáceres não apresentou os maiores valores dos índices e Nossa Senhora do Livramento e Santo Antônio do Leverger foram os que sobressaíram.

É relevante considerar a questão temporal nos estudos envolvendo a dengue, pois esta é uma doença persistente por apresentar diversos fatores envolvidos, como a dinâmica de transmissão do patógeno no hospedeiro invertebrado e vertebrado, a resposta imune dos hospedeiros e os aspectos ambientais que favorecem o desenvolvimento do vetor. Isso faz com que, em determinados períodos, haja ou não maior transmissão do vírus e, consequentemente, maior incidência da doença em diferentes anos e distintas localidades.

A autocorrelação de Moran Global espacial mostrou que existe fraca dependência dos municípios com relação aos índices avaliados, porém a autocorrelação de Moran Local indicou os locais que apresentaram agrupamentos, isto é, indicou as áreas que devem ser investigadas/monitora- 
das com maior atenção, de acordo com cada índice apontado. A situação dos municípios estudados, localizados na porção sul do Estado, pode estar contribuindo para a presença e transmissão da doença, uma vez que apresentam disparidades econômicas e sociais consideráveis.

Neste estudo, os procedimentos adotados basearam-se em dados de notificação de casos (confirmados), que podem ser utilizados para a vigilância e o controle da dengue, por meio de um planejamento de ações, já que existem os dados das unidades de maior risco, identificadas nos diversos períodos, ou seja, as áreas do município que devem ser priorizadas quanto à vigilância e ao controle dos vetores e da doença. Além disso, esses dados podem auxiliar, por exemplo, os profissionais destinados à aplicação de substâncias químicas nos locais de maior incidência. A partir da operacionalização dos índices, é possível projetar cenários relativos aos riscos da endemia, como a associação da verificação entomológica (a utilização de armadilhas para as formas imaturas e adultas do vetor da dengue), os Sistemas de Informação Geográfica e as técnicas cartográficas.

Tudo isso pode contribuir para a escolha de diferentes metodologias a serem aplicadas no combate e controle da doença. No entanto, a questão socioambiental deve ser analisada e as políticas públicas devem estar voltadas para as questões da interação entre o ambiente, o ser humano, o vetor da doença e patógeno, considerando a desigualdade social. Isso porque a dengue, muitas vezes tratada com negligência, não prevalece só em condições de pobreza, mas também contribui para a manutenção do quadro de desigualdade, que representa forte entrave ao desenvolvimento do país. Diante desse contexto, pode-se considerar que ainda há muito a ser estudado em relação à epidemiologia da dengue no Brasil e, sobretudo, em municípios do bioma Pantanal.

\section{Agradecimentos}

Agradecemos à Secretaria Regional de Saúde de Cáceres e à Vigilância Epidemiológica de Cuiabá pela disponibilização dos dados utilizados nesta pesquisa. Ao Projeto Modelagem de indicadores ambientais para a definição de áreas prioritárias e estratégicas à recuperação de áreas degradadas da região sudoeste de Mato Grosso, vinculado à sub-rede de estudos sociais, ambientais e de tecnologias para o sistema produtivo na região sudoeste mato-grossense REDE ASA - financiada pelo Edital MCT/CNPq/FNDCT/FAPs/MEC/CAPES/ PRO-CENTRO-OESTE N. 031/2010. 


\section{Referências}

ANSELIN, L. Local indicators of spatial association (LISA). Geographical Analises, v. 27, n. 2, p. 93-115, 1995.

BARBOSA, G. L.; LOURENÇO, R. W. Análise da distribuição espaço-temporal de dengue e da infestação larvária no município de Tupã, Estado de São Paulo. Revista da Sociedade Brasileira de Medicina Tropical, v. 43, n. 2, p. 145-151, 2010.

BRASIL. Ministério da Saúde. Secretaria de Vigilância em Saúde. Informe Epidemiológico da Dengue: análise de situação e tendências, 2010.

COX, J. et al. Habitat segregation of dengue vectors along an urban environmental gradient. The American Journal of Tropical Medicine and Hygiene, v. 76, n. 5, p. 820-826, 2007.

CRONER, C. M.; SPERLING J.; BROOME, F. R. Geographic information systems (GIS): new perspectives in understanding human health and environmental relationships. Statistics in Medicine, v. 15, p. 1961-1977, 1996.

CUNHA, R. V.; ATANAKA-SANTOS, M. Prioridades da pesquisa em epidemiologia na região do Pantanal brasileiro. Cadernos de Saúde Pública, v. 27, n. 6, p. 10501051, 2011.

FORATTINI, O. P. Culicidologia médica: identificação, biologia e epidemiologia. São Paulo: Universidade de São Paulo, 2002. 545p.

GALLI, B.; CHIARAVALLOTI NETO. F. Modelo de risco tempo-espacial para identificação de áreas de risco para ocorrência de dengue. Revista de Saúde Públi$c a$, v. 42, n. 4, p. 656-663, 2008.

GLASSER, C. M.; GOMES, A. C. Clima e sobreposição da distribuição de Aedes aegypti e Aedes albopictus na infestação do Estado de São Paulo. Revista de Saúde Pública, v. 36, n. 2, p. 166-172, 2002.

IBGE. Instituto Brasileiro de Geografia e Estatística. Censo 2010. Disponível em: <http://www.ibge.gov.br/home/estatistica/populacao/censo2010/>. Acesso em: 23 ago. 2011.

MARQUES, A. J. Aplicações de técnicas de autocorrelação espacial para avaliação da organização das habitações nas mesorregiões do estado do Paraná. Observaotrium: Revista Eletrônica de Geografia, v. 1, n. 3, p. 89-105, 2009.

MENDONCA, F. A. Aquecimento global e saúde: uma perspectiva geográfica notas introdutórias. Revista Terra Livre, São Paulo, n. 20, p. 205-221, 2003.

MUKAI, A. O.; ALVES, K. S C.; NASCIMENTO, L. F. C. Análise espacial das internações por pneumonia na região do Vale do Paraíba (SP). Jornal Brasileiro de Pneumologia, v. 35, n. 8, p. 753-758, 2009.

REBÊLO, J. et al. Distribuição de Aedes aegypti e da dengue no Estado do Maranhão, Brasil. Cadernos de Saúde Pública, v. 15, n. 3, p. 477-486, 1999.

RIBEIRO, A. F. et al. Associação entre incidência de dengue e variáveis climáticas. Revista de Saúde Pública, v. 40, n. 4, p. 671-676, 2006. 
SIQUEIRA-JUNIOR, J. B. et al. Spatial point analysis based on dengue surveys at household level in central Brazil. Biomed Central Public Health, v. 8, n. 361, p. 1-9, 2008.

SOUZA, C. K. J. et al. Análise espacial da dengue no município brasileiro de Cáceres/MT, no ano de 2009. In: $3^{\circ}$ Simpósio de Geotecnologias no Pantanal. Anais... Cáceres: Embrapa/INPE, p. 999-1009, 2010.

TEIXEIRA, M.G. et al. Dynamics of dengue virus circulation: a silent epidemic in a complex urban área. Tropical Medicine and International Health, v. 7, n. 9, p. 757-762, 2002.

WEN, T. H. et al. Spatial mapping of temporal risk characteristics to improve environmental health risk identification: A case study of a dengue epidemic in Taiwan. Science of the Total Environment, v. 367, p. 631-640, 2006.

Rosilainy Surubi Fernandes - Possui Graduação em Ciências Biológicas pela Universidade do Estado de Mato Grosso e Mestrado em Ciências Ambientais pela mesma Universidade. Atualmente é doutoranda pelo programa de Pós-Graduação em Biologia Parasitária do Instituto Oswaldo Cruz.

Sandra Mara Alves da Silva Neves - Possui Graduação em Geografia pela Universidade Federal de Mato Grosso do Sul, Mestrado em Geografia pela Universidade Estadual Paulista Júlio de Mesquita Filho e Doutorado em Ciências (Geografia) pela Universidade Federal do Rio de Janeiro. Atualmente é professora da Universidade do Estado de Mato Grosso.

Ronaldo José Neves - Possui Graduação em Geografia pela Universidade Federal de Mato Grosso do Sul. Mestrado em Geografia pela Universidade Estadual de Maringá e Doutorado em Ciências (Geografia) pela Universidade Federal do Rio de Janeiro. Atualmente é professor da Universidade do Estado de Mato Grosso.

Recebido para publicação em 30 de outubro de 2013

Aceito para publicação em 19 de janeiro de 2014 